\title{
Etude de la fixation des pesticides organophosphorés sur les constituants protéiques du lait
}

\author{
par \\ Victoire RAKOTOVELO*, J.C. LHUGUENOT*, \\ D. LORIENT*
}

\section{Ré s u m é}

Les pesticides organophosphorés sont instables à $\mathrm{pH}$ acide. Leur intégrité observée au niveau du foie suppose donc un rôle protecteur des constituants alimentaires tels que les protéines et/ou les lipides, au cours du transit digestif.

La première étape de cette étude consiste à vérifier la fixation de 3 pesticides organophosphorés sur des protéines du lait; ce dernier, constituant un bon modèle en alimentation humaine. Ces pesticides sont le parathion (0,0-diéthyl 0-(p-nitrophényl) phosphorothioate), le malathion (0,0-diméthyl S-[1,2-di (éthoxycarbonyl) éthyl] phosphorodithioate), le trithion [0,0-diéthyl S-(4-chlorophényl-thiométhyl) phosphorothiolothionate] ou carbophénothion.

Les études de fixation des pesticides sur les protéines du lait ont été réalisées par dialyse d'équilibre. La quantité fixée est déterminée par dosage du pesticide résiduel dans la solution de contre-dialyse, en tenant compte de la dégradation de ce pesticide dans les mêmes conditions. Cette détermination est effectuée par chromatographie en phase gazeuse (colonne capillaire et détection par capture d'électrons avec source de $\mathrm{Ni}^{63}$ ). La quantité minimale mesurable est de l'ordre de $10 \mathrm{pg}$ pour les pesticides organophosphorés étudiés.

Une étude comparative de la fixation des 3 pesticides à $\mathrm{pH}$ voisin de 7, sur des susbtrats protéiques dérivés du lait tels que la caséine entière et différents types de lactosérum (entier, délactosé, déminéralisé) a montré que les 3 pesticides se fixent de façon significative sur

* Département de Biochimie Alimentaire (ENSBANA), Campus Universitaire, 21100 Dijon. 
la caséine $(0,02$ à $0,21 \mu \mathrm{g} / \mathrm{mg}$ de protéine) et plus faiblement sur les autres substrats. Leur comportement vis-à-vis des protéines pures dépend étroitement du $\mathrm{pH}$, les quantités fixées variant selon les différentes caséines utilisées. Les propriétés fixatrices de la caséine sont sans doute en relation avec la présence de nombreux résidus séryle et phosphoséryle, par analogie au site porté par la cholinestérase.

Ces résultats sont à rapprocher de ceux obtenus par Freed et al. (1979) en ce qui concerne le "protein-binding " du parathion et du malathion sur un extrait d'homogénat de foie.

\section{Mots clés Malathion - Trithion - Carbophénothion. \\ Titre abrégé \\ Fixation des pesticides OP sur des protéines du lait. \\ S u m m a ry \\ MILK PROTEINS AS BINDING PROTEINS FOR ORGANOPHOSPHORUS PESTICIDES}

Fixation, pesticides organophosphorés - Protéines du lait - Parathion -

It is well known that organophosphorus pesticides degrad rapidly in acid medium. According to Metcalf (1971), their observed toxicity depends upon two types of molecules: original pesticide and bioactivated molecule. The latter may be obtained only with liver multifonction oxidase (MFO) enzymes and cytochrome P 450. This suggests that a protective mechanism by interaction with proteins and/ or lipids allows the pesticides to pass without change through the digestive tract, and oxidation by MFO enzyme only takes place after the pesticide reaches the liver.

The first stage of this study was to verify the binding of three organophosphates pesticides with milk proteins, as milk is a well balanced human food material.

The three organophosphorus pesticides used in this study were: parathion (0,0-diethyl 0-( $p$-nitrophenyl) phosphorothioate), malathion (0,0-dimenthyl $S$-[1,2-di (ethoxycarbonyl) ethyl] phosphorodithioate), trithion [0,0-diethyl S-(4-chlorophenyl-thiomethyl) phosphorothiolothionate].

The amount of pesticide bound by the milk proteins was found from the difference between the concentrations in the solutions outside the dialysis membrane in similar trials where milk protein was present, or absent, inside the dialysis membrane. This also allowed for any degradation of pesticide occurring during the experimental period. 
Pesticide determinations were carried out using capillary column gas-liquid chromatography (electron capture detection with $\mathrm{Ni}^{6 s}$ as the radioactivity source).

A comparative study of the binding of the three pesticides, at pH 6.6 on protein substrates derived from milk, has shown that they were strongly bound by casein, (0.02 to $0.21 \mu \mathrm{g} / \mathrm{mg}$ protein) and bound less strongly by the other proteins studied (delactosed, demineralized or whole lactoserum). A similar mechanism may be proposed for the binding of pesticides with casein i.e. dependence on the presence of seryl and phosphoseryl groups in the protein molecule as is known to be the case for pesticide binding to the enzyme cholinesterase.

Our results must be compared with those of Freed et al. (1979) who studied the binding of organophosphorus molecules with liver tissue extract.

Key words

Protein-binding - Milk proteins - Organophosphates - Malathion - Parathion Trithion - Carbophenothion.

\section{INTRODUCTION}

L'utilisation des pesticides organophosphorés (OP) dans le domaine agricole s'est développée ces dernières décades aux dépens de celle des organochlorés, du fait de leur faible rémanence. Cependant, certains dérivés des composés organophosphorés sont plus toxiques et plus persistants que le composé qui leur a donné naissance. D'après Metcalf (1971), chez le rat, le paraoxon produit d'oxydation du parathion, est 150 fois plus toxique que ce dernier par voie orale. D'où l'intérêt de se préoccuper du devenir des pesticides OP apportés par l'alimentation humaine, au cours du transit digestif.

Pour cela nous avons pensé à un rôle protecteur des constituants alimentaires. L'oxydation des phosphorothionates sous l'action des enzymes appartenant au groupe des MFO (multifonction oxidase) en présence du cytochrome $\mathrm{P} 450$ se passant au niveau du foie, et ces dérivés oxydés étant responsables de la toxicité des composés organophosphorés d'après ce que nous avons dit précédemment, les pesticides organophosphorés n'ont pu traverser le tube digestif où sont réunis les facteurs favorisant leur dégradation ( $\mathrm{pH}$ acide, humidité, température $37^{\circ} \mathrm{C}$ au niveau de l'estomac, puis $\mathrm{pH}$ basique au niveau de l'intestin), tout en conservant leur intégrité, que dans la mesure où ils ont bénéficié d'un rôle protecteur de certains constituants alimentaires.

C'est ainsi que nous avons été amenés à vérifier la fixation des pesticides OP sur des substrats protéiques d'origine laitière, le lait et les produits laitiers occupant une part prépondérante dans l'ali- 
mentation humaine; certains d'entre eux pouvant d'ailleurs être mis en contact avec des pesticides OP provenant de fruits incorporés comme le cas des yaourts aux fruits.

Nos premières études ont déjà montré que les principaux constituants du lait (protéines, matières grasses), réduisent le taux de dégradation des pesticides OP tel qu'on peut l'observer dans l'eau (cf. fig. 1) (V. Rakotovelo, 1981). La fixation des pesticides OP sur les protéines a été étudiée par Freed (1979) sur un homogénat de foie, le foie étant un organe de détoxication de ces composés. Il est ressorti de leur étude que les composés OP peuvent se lier à des protéines autres que la cholinestérase dont ils sont les inhibiteurs spécifiques, et que cette liaison, bien que de faible importance quantitativement et de nature non définie, peut conduire à une persistance des pesticides.

\section{MATERIEL ET METHODES}

\section{Matériel}

- Les pesticides OP utilisés sont des solutions standards de pureté supérieure à $99 \%$.

- Tous les réactifs sont exempts de résidus : acétone, méthanol, éther de pétrole $40-60^{\circ} \mathrm{C}$, hexane.

- Toutes les pièces de verrerie sont lavées à l'aide d'un détergent, rincées à l'eau distillée puis à l'acétone exempt de résidu, avant d'être placées à l'étuve à $100^{\circ} \mathrm{C}$ pendant au moins $14 \mathrm{~h}$.

Les échantillons ont été analysés par chromatographie gaz-liquide à l'aide d'un chromatographe Packard 417, bicolonne, équipé d'un détecteur à capture d'électrons à fréquence variable, avec source de $\mathrm{Ni}^{63}(10 \mathrm{mc})$.

L'appareil est équipé :

- d'une colonne capillaire en pyrex, de longueur $25 \mathrm{~m}$, de diamètre intérieur $0,4 \mathrm{~mm}$, à phase stationnaire OV 1 (Spiral-Dijon);

- d'un injecteur de Ros (Spiral) ;

- d'un intégrateur-enregistreur Hewlett-Packard.

\section{Méthodes}

\subsection{Dialyse d'ÉQUilibre}

La méthode de fixation des pesticides organophosphorés sur les protéines du lait découle de celle de Freed. $1 \mathrm{ml}$ de solution protéique à $5 \%$ est placé dans un tube à dialyse de $6 \mathrm{~mm}$ de diamètre lui-même plongé dans $10 \mathrm{ml}$ de solution tampon de contre-dialyse (tampon phosphate $\mathrm{pH}$ 7) contenant le pesticide à $1 \mathrm{\mu g} / \mathrm{ml}$. La dialyse s'effectue sous agitation constante à température ambiante $\left(20^{\circ} \mathrm{C}\right)$ pendant $15 \mathrm{~h}$. 


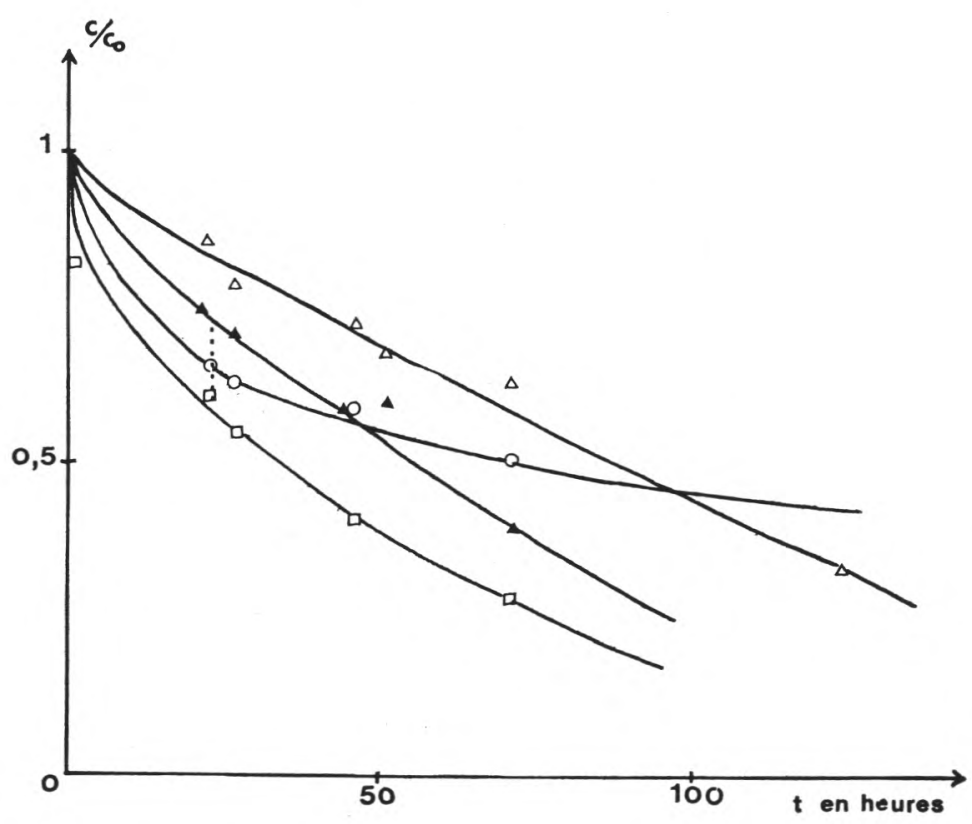

LEG ENDE

Ecart-type
Lait entier
Lait demi-écrémé
Lait êcrểé
Eau distillée

fig. 1

Etude de l'influence de la teneur en matière grasse du lait sur la dégradation du trithion.

Influence of fat content on trithion degradation in milk.

Chaque essai est effectué en double et l'addition d'un standard interne OP (parathion ou méthylparathion selon le comportement chromatographique), permet l'évaluation du rendement des opérations analytiques.

\subsection{Extraction}

Après dialyse, les pesticides OP ont été extraits du liquide de contre-dialyse suivant la méthode mise au point au laboratoire par 
Humbert et Bellinaso (1976-1977). Cette méthode consiste en une extraction par partage liquide-liquide, séchage sur colonne de sulfate de sodium anhydre, puis concentration de l'extrait ainsi obtenu.

\subsection{Chromatographie GaZ-Liquide}

La température du four variait entre 160 et $220^{\circ} \mathrm{C}$ suivant le composé analysé. L'appareil nous permet de détecter des quantités de l'ordre de $10 \mathrm{pg}$ pour les pesticides étudiés.

\section{RESULTATS ET DISCUSSION}

Les premiers résultats ont été obtenus en plaçant simultanément dans le liquide de contre-dialyse les 3 pesticides OP: parathion, malathion et trithion. La quantité de pesticides fixée par la protéine est obtenue par différence entre la quantité introduite avant dialyse et celle résiduelle, en tenant compte de la dégradation du même pesticide dans les mêmes conditions.

\section{TABLEAU 1 - TABLE 1}

Etude de la fixation des pesticides organophosphorés sur des protéines du lait (Quantité fixée en $\mu \mathrm{g} / \mathrm{mg}$ de protéine)

Binding of organophosphorus pesticides with milk proteins

\begin{tabular}{l|c|c|c}
\hline \multicolumn{1}{r|}{ Pesticides } & Malathion & Parathion & Trithion \\
Protéines & & 0,020 & 0,210 \\
\hline Caséine entière & & 0,020 & 0,300 \\
Lactosérum entier & \multirow{2}{*}{0,013} & & 0,310 \\
Protéines du lactosérum 80\% & & 0,250 \\
Protéines du lactosérum 37\% & & 0,250 \\
Lactosérum délactosé & & 0,210 \\
Lactosérum lactosé déminéralisé & & 0,110 \\
$\beta$-lactoglobuline & & \\
\hline
\end{tabular}

D'après ces résultats (cf. tableau 1 et fig. 2) nous avons constaté que les quantités de composés $\mathrm{OP}$ retenues par les protéines du lait sont inférieures à celles observées par Freed pour l'extrait d'homogénat de foie. 

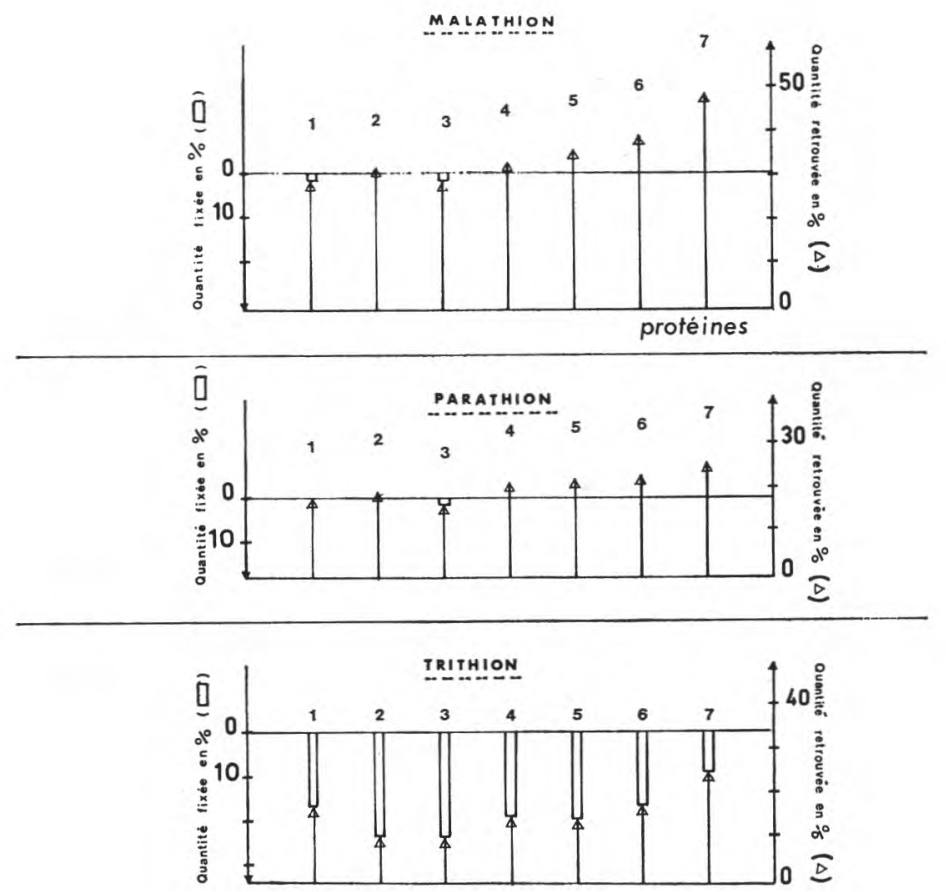

LEGENDE

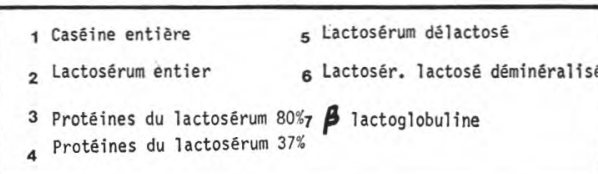

fig. 2

Etude de la fixation des pesticides organophopshorés sur des protéines du lait.

Binding of organophosphorus pesticides with milk proteins.

Seul le trithion, de structure différente des deux autres (chloré), se fixe sur toutes les protéines étudiées.

Le malathion et le parathion, au contraire, ne se fixent qu'en très faible quantité, et uniquement sur la caséine dans le cas du parathion. L'analogie de l'allure générale des 3 graphiques obtenus indique :

- d'une part l'éventualité d'un effet de compétition puisque l'on observe une fixation préférentielle du trithion;

- d'autre part un effet protecteur des protéines sur la dégradation du parathion et du malathion. 
A la suite de ces résultats préliminaires, il s'est avéré nécessaire d'étudier le comportement de chaque composé OP par rapport aux protéines mises en cause.

Nous avons alors repris l'expérience en étudiant l'influence du $\mathrm{pH}$ sur la fixation du malathion et du trithion par la caséine (cf. fig. 3). Nous avons pu démontrer que moyennant certaines conditions de $\mathrm{pH}$ :

- le malathion peut aussi se fixer sur la caséine ;

- que le comportement du pesticide vis-à-vis des protéines pures dépend étroitement du $\mathrm{pH}$. Pour le malathion, l'optimum de fixation semble se situer aux environs de $\mathrm{pH} 4,5$, pour le trithion, aux environs de $\mathrm{pH} 5$;

- les quantités maximales de pesticide fixées par mg de caséine ont été respectivement de 0,15 et $0,11 \mu \mathrm{g}$ pour le malathion et le

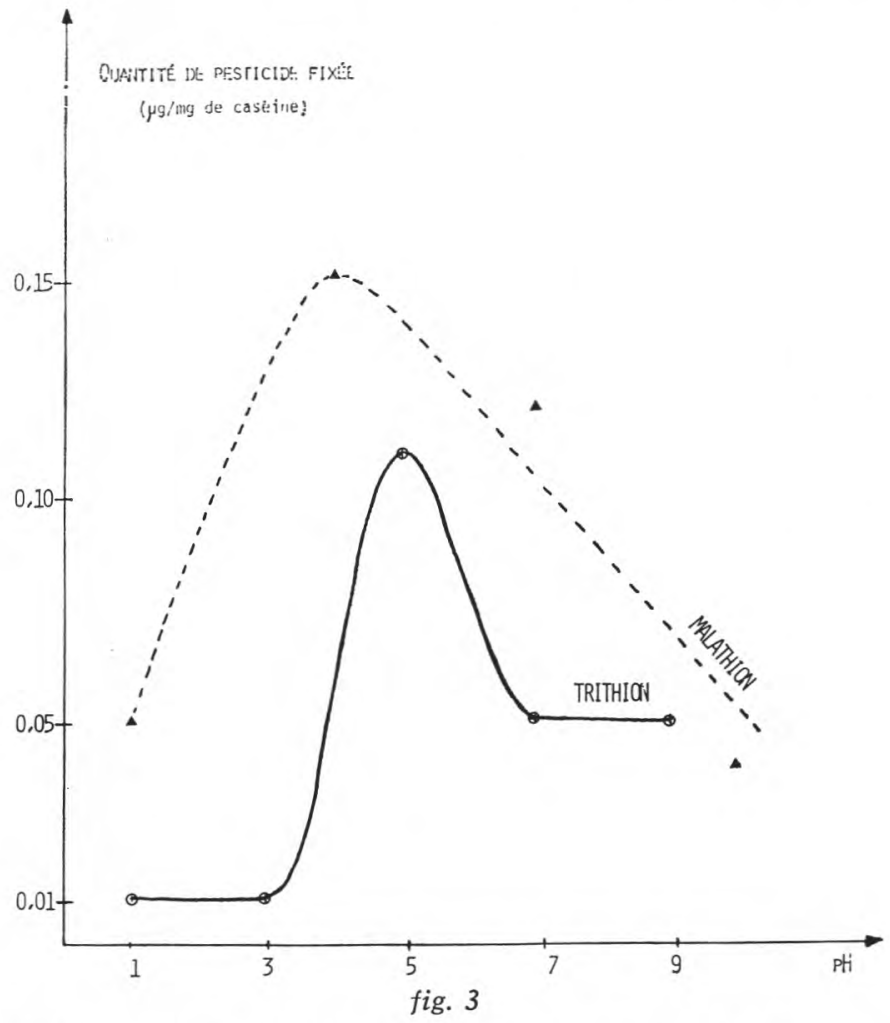

Influence du $\mathrm{pH}$ sur la fixation du malathion et du trithion par la caséine bovine.

Effect of pH on malathion and Carbophenothion binding with casein. 
trithion; ainsi, la fixation des 2 pesticides est différente selon que ceux-ci sont placés seuls ou simultanément dans le liquide de contredialyse.

Par ailleurs, des essais d'inhibition de la cholinestérase par contact avec le malathion et le trithion ont été réalisés. Des prélèvements au cours du temps du pesticide résiduel ont montré que, dans tous les cas, les quantités de pesticide libre retrouvées après extraction des solutions protéiques, sont supérieures à celles trouvées dans les solutions témoins, ce qui montre bien un effet protecteur des protéines sur la dégradation des pesticides OP (Borde, résultats non publiés).

Deux types d'expériences complémentaires paraissent ainsi nécessaires pour étudier les interactions pesticides-protéines :

- fixation des pesticides par dialyse d'équilibre;

- effet protecteur des protéines par mise en contact direct puis extraction du pesticide.

Des études complémentaires des différents paramètres de fixation pourront nous renseigner sur la nature des liaisons protéinepesticide. De plus, l'étude de la fixation des pesticides sur des phosphoprotéines comme la phosvitine et la vitelline nous permettront de rapprocher les propriétés fixatrices de la caséine à la présence de nombreux résidus séryle et phosphoséryle, par analogie au site actif de la cholinestérase.

En conclusion, les résultats présentés ci-dessus posent plus de problèmes qu'ils n'en résolvent; c'est pourquoi nous n'avons pas la prétention de clore ce chapitre.

\section{Remerciements}

Nous remercions vivement $\mathrm{M}$. le $\mathrm{Pr}$ G. Lavoué pour les précieux conseils et la collaboration qu'il nous a prodigués lors de la réalisation de cette étude.

\section{Bibliographie}

Bellinaso (C.) (1977). - Contribution à la détermination de la teneur en pesticides organochlorés dans les fruits sur sucre destinés à l'incorporation dans les produits laitiers. Mémoire ENSBANA, Université de Dijon.

BULL (D. L.) (1972). - Metabolism of organophosphorus insecticides in animals and plants. Residue Review, 43, 1-21.

BYron et al. (1978). - Fundamentals of dairy chemistry. 2nd Edition.

FREED (V.H. et al.) (1979). - Physical chemical properties of several organophosphates: some implication in environment and biological behavior. Pesticide Biochem. Physiol., 10, 203-211.

Hedin (P. A.) (1982). - New concepts and trends in pesticide chemistry. J. Agric. Food Chem., $10, \mathrm{n}^{\circ} 2$. 
MetCalf (R. L.) (1971). - The chemistry and biology of pesticides. Pesticides in the environment, 1, part I. Ed. R. White-Stevens.

RaKotovelo (Victoire) (1981). - Contribution à l'étude de la dégradation des pesticides organophosphorés. Influence de la matière grasse et de la fraction protéique du lait. Mémoire ENSBANA, Université de Dijon.

Roudot (F.) (1980). - Etude de la dégradation des pesticides organophosphorés. Influence de la teneur en eau, de la température et de la teneur en matière grasse. Mémoire ENSBANA, Université de Dijon. 